\title{
Increased incidence of cytomegalovirus but not Chlamydia pneumoniae in atherosclerotic lesions of arteries of lower extremities from patients with diabetes mellitus undergoing amputation
}

\author{
T-M Lin, W-i Chen, H-Y Chen, P-W Wang, H-L Eng
}

See end of article for authors' affiliations

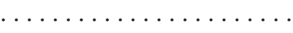

Correspondence to: Dr H-L Eng, Department of Pathology, Chang Gung Memorial Hospital, 123 Ta-pei Road, Niao-Sung Hsiang, Kaohsiung,

Taiwan;

eng6166@ms8.hinet.net

Accepted for publication 15 January 2003

\begin{abstract}
Aims: To evaluate the association between cytomegalovirus (CMV) or Chlamydia pneumoniae infection and the development of accelerated atherosclerotic lesions in patients with diabetes who are known to have an impaired immune response to infection and a high incidence of atherosclerosis.

Methods: Two hundred arterial samples from patients with diabetes who had undergone surgical amputation for gangrenous lower limbs were selected to assess the presence of CMV or C pneumoniae nucleic acid by means of the polymerase chain reaction.

Results: CMV nucleic acid sequences were detected in 64 of $200(32 \%)$ samples and C pneumoniae in seven of $200(3.5 \%)$ arterial samples with severe atherosclerosis. Of those positive for $C$ pneumoniae, six were also positive for CMV.

Conclusion: The significantly higher incidence of CMV nucleic acid sequences in the arterial samples of patients with diabetes supports the hypothesis that this organism is involved in the pathogenesis of atherosclerosis in patients with diabetic mellitus. It is possible that the potential role of different infectious agents in the pathogenesis of atherosclerosis might rely on their biological properties and their infectivity in hosts with varying immunological status.
\end{abstract}

$\mathrm{P}$ atients with diabetes are relatively immunocompromised, remain persistently at risk for infectious complications, ${ }^{12}$ and are highly prone to develop accelerated atherosclerosis. ${ }^{34}$ The widely accepted "response to injury" hypothesis of atherosclerosis proposed that an initial insult to the arterial wall triggers atherogenesis and plaque formation. ${ }^{5}$ Although many factors may initiate atherogenesis, the process ultimately involves an inflammatory state in which macrophages and $\mathrm{T}$ cells play a major role. ${ }^{6-8}$ Antigen within the plaque may trigger/perpetrate the immunological activity and therefore the atherogenic inflammation. The potential role of common infectious agents in the pathogenesis and progression of atherosclerosis has been studied increasingly over the past decade. Herpes simplex virus and cytomegalovirus (CMV) were first detected in human atheromatous tissue by the groups of Melnick and Benditt, respectively, in 1983..$^{10}$ Antigens and nucleic acid sequences of CMV have been found in arterial lesions in human atherosclerosis, ${ }^{11}$ and several studies have also shown an association between atherosclerosis and antibodies against CMV. ${ }^{12}$ However, the most convincing evidence for a role for herpes viruses in atherosclerosis comes mainly from the association of CMV with coronary artery disease in transplant accelerated atherosclerosis ${ }^{13-15}$ and restenosis following angioplasty. ${ }^{16}$ The evidence for $C$ pneumoniae as a potential causative agent is based on the findings from numerous sero-epidemiological studies, ${ }^{17}$ the examination of atheromatous plaque specimens, ${ }^{18}$ animal models, ${ }^{19}$ and recently pilot anti-chlamydial antibiotic intervention trials. ${ }^{20}{ }^{21}$ Despite a high frequency of antibody against $C$ pneumoniae in the population, these studies have consistently demonstrated a significant difference between earlier C pneumoniae infection and atherosclerosis.

\footnotetext{
"Several studies have also shown an association between atherosclerosis and antibodies against cytomegalovirus"
}

We hypothesise that the impaired immune response in patients with diabetes may cause the reactivation of persistent pathogens, such as CMV and C pneumoniae, resulting in damage to the vessel wall, and thus initiating atherosclerosis. There has been only one report evaluating the role of CMV infection in the development of atherosclerosis in patients with diabetes. In that study, Visseren and co-workers ${ }^{22}$ detected anti-CMV antibodies more often in those patients with diabetes who had atherosclerosis compared with those without atherosclerosis $(70.7 \% v 45.2 \%)$.

The purpose of our study was to evaluate the association between CMV or C pneumoniae infection and the development of accelerated atherosclerotic lesions in patients with diabetes by the detection of CMV and C pneumoniae nucleic acids by means of the polymerase chain reaction (PCR) in atherosclerotic tissues from arterial samples obtained from patients with diabetes.

\section{MATERIALS AND METHODS \\ Patients}

To study the presence of CMV and C pneumoniae in the arterial wall, archival, paraffin wax embedded arterial tissue samples (including those of the anterior and posterior tibial arteries, and the pedis dorsalis artery) of 200 patients with type II diabetes mellitus for more than five years, who had undergone surgical amputation of lower extremities between 1990 and 1996 in Kaohsiung Medical Center, Chang Gung Memorial Hospital, Taiwan, were selected. They included 119 men and 81 women, with a mean age of 65 years and 69 years, respectively. In addition, 24 non-diabetic patients who had undergone surgical amputation of a lower extremity as a result of other

Abbreviations: CMV, cytomegalovirus; IE, immediate-early gene; PCR, polymerase chain reaction 
Table 1 Sequences of primers used

\begin{tabular}{|c|c|c|}
\hline Primers & Region & Nucleotide sequence \\
\hline \multicolumn{3}{|l|}{ CMV } \\
\hline \multicolumn{3}{|l|}{ Outer PCR } \\
\hline CMVR-1 & IE exon 4 & GTAATGAAGCGCCGCATTGAGGAGA \\
\hline CMVL-1 & IE exon 4 & GGCAACTTCCTCTATCTCAGACAC \\
\hline \multicolumn{3}{|l|}{ Inner PCR } \\
\hline CMVR-2 & IE exon 4 & CCTAGTGTGGATGACCTACGGGCCA \\
\hline CMVL-2 & IE exon 4 & CAGACACAGTGTCCTCCCGCTCCTC \\
\hline \multicolumn{3}{|c|}{ Chlamydia pneumoniae } \\
\hline $\mathrm{HL}-1$ & & GTTGTTCATGAAGGCCTACT \\
\hline HR-1 & & TGCATAACCTACGGTGTGTT \\
\hline \multicolumn{3}{|r|}{ 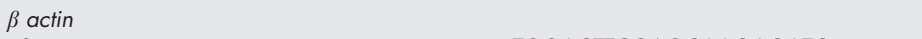 } \\
\hline$\beta 3$ & & TGGACTTCGAGCAAGAGATG \\
\hline$\beta 4$ & & СTCCTTCTGCATCCTGTC \\
\hline
\end{tabular}

causes, such as traumatic injury, were also collected as a control patient group. The histological slides were reviewed by a pathologist and appropriate paraffin wax embedded tissue blocks were sectioned for PCR analysis for both CMV and C pneumoniae.

\section{Detection of CMV and C pneumoniae nucleic acid Tissue preparation}

Archival arterial wall tissue had been fixed in 10\% buffered formalin and embedded in paraffin wax. Ten $5 \mu \mathrm{m}$ thick paraffin wax embedded sections were cut using disposable knives to avoid contamination between blocks. DNA was purified from tissue sections using the Chelex boiling method. Briefly, sections were boiled in a $25 \%$ suspension of Chelex 100 chelation resin (Sigma Chemical Co, St Louis, Missouri, USA) in sterile water. The resulting supernatants were extracted with phenol/chloroform by standard methods and precipitated with ethanol. The DNA pellets were resuspended in $50 \mu \mathrm{l}$ Tris/ $\mathrm{HCl}$ buffer at $\mathrm{pH}$ 8.0. Mock extractions of buffer were done and amplified to ensure that no contamination occurred. DNA extraction and PCR amplification were performed in separate rooms with a different pipette with aerosol resistant tips.

\section{Controls}

The positive CMV control was prepared in human embryonic fibroblast (HEF) cells infected with CMV strain AD 169 in a $75 \mathrm{~cm}^{2}$ flask. After a strong cytopathic effect had developed, the cell layer was washed with phosphate buffered saline. The cells were scraped into $5 \mathrm{ml}$ PBS, and the suspension was frozen and thawed three times, followed by boiling for 10 minutes. The cellular debris was removed by centrifugation at $2000 \times g$. The supernatant was stored at $-70^{\circ} \mathrm{C}$. Stock titres were determined by plaque assay.

The positive C pneumoniae control was prepared in HL cells infected with C pneumoniae strain AR-39 in a $75 \mathrm{~cm}^{2}$ flask for 72 hours. The cells were scraped into $1.0 \mathrm{ml}$ sucrose phosphate glutamic acid medium. The titres of these preparations were determined by the method of Furness et al. ${ }^{23}$

The CMV and C pneumoniae controls were added to lysis buffer (100mM Tris/HCl, pH 8.0, 25mM EDTA, 2\% Triton $\mathrm{X}-100$ ) with $50 \mu \mathrm{g} / \mathrm{ml}$ proteinase $\mathrm{K}$. The lysate was incubated overnight at $50^{\circ} \mathrm{C}$, and extracted sequentially with phenol, phenol/chloroform (1/1), and chloroform/isoamyl ethanol (24/1). The aqueous phase was precipitated with one tenth of $3 \mathrm{M}$ ammonium acetate ( $\mathrm{pH} 5.2$ ), one hundredth $10 \%$ glycogen, and two volumes of absolute ethanol. DNA pellets were air dried and resuspended in $50 \mu \mathrm{l}$ Tris buffer.

Primers and probes

Table 1 lists the primers and probes. For the detection of CMV nucleic acid by nested PCR, primers derived from the immediate-early gene (IE) exon 4 were used. ${ }^{24}$ For the detection of $C$ pneumoniae nucleic acid, DNA samples were amplified using $C$ pneumoniae specific primers HL- 1 and HL-2. ${ }^{25}$ In all experiments, the DNA extracts were also amplified with $\beta$ actin primers $\beta 3$ and $\beta 4$ as controls for DNA preservation and the exclusion of false negative results.

\section{PCR for CMV}

A $50 \mu \mathrm{l}$ volume of reaction mixture was prepared containing $1 \mathrm{mmol} /$ litre each of dATP, dCTP, dGTP, and dTTP; $25 \mathrm{pmol}$ of each oligonucleotide primer; $0.5 \mathrm{U}$ of Taq polymerase (Perkin Elmer, Oak Brook, Illinois, USA); and $5 \mu \mathrm{l}$ of specimen DNA in a buffer solution. The buffer solution consists of $50 \mathrm{mM} \mathrm{KCl}$, $3 \mathrm{mM} \mathrm{MgCl}{ }_{2}, 10 \mathrm{mM}$ Tris/HCl, pH 9.0, and 0.1\% Triton X-100. Amplification of the target portion of the CMV IE gene was achieved by means of a nested PCR using a CMVR-1/CMVL-1 primer set in the first and a CMVR-2/CMVL-2 primer set in the second PCR. Samples were amplified using 35 cycles of denaturation at $94^{\circ} \mathrm{C}$ for one minute, primer annealing at $55^{\circ} \mathrm{C}$ for two minutes, and chain extension at $72^{\circ} \mathrm{C}$ for three minutes in a Perkin-Elmer Cetus thermocycler. PCR products ( $10 \mu \mathrm{l})$ were separated by electrophoresis using $1.5 \%$ agarose gel and visualised by ethidium bromide staining. To determine the sensitivity of the PCR procedures in detecting CMV DNA, varying amounts of DNA ( $10^{2}$ to $10^{3}$ plaque forming units) from the positive control DNA were amplified. Distilled water was used as a negative control in each set of PCR reactions.

\section{PCR for C pneumoniae}

PCR was performed as described by Campbell and colleagues $^{25}$ to detect $C$ pneumoniae specific DNA. Negative and positive controls consisting of PCR reagents but lacking the specimen, and varying dilutions of purified $C$ pneumoniae DNA, respectively, were included in each PCR run. The amplification products, the expected $437 \mathrm{bp} C$ pneumoniae specific DNA sequences, were analysed by gel electrophoresis through a $1.0 \%$ agarose gel according to standard protocols and transferred by Southern blotting to a nylon membrane (Amersham Life Science, Little Chalfont, Buckinghamshire, UK). The products were confirmed by hybridisation to a digoxigenindUTP labelled probe. DNA probes were labelled by means of the Genius DNA labelling and detection kit (Boehringer Mannheim Biochemicals, Indianapolis, Indiana, USA), and hybridisation was detected by immunochemiluminescence using Lumiphos 530, according to the manufacturer's directions.

\section{Statistical analysis}

Statistical analysis was carried out by Fisher's exact tests. Results were considered significant when $\mathrm{p}<0.05$. 
Table 2 Cytomegalovirus (CMV) and Chlamydia pneumoniae DNA in vessel wall of patients with diabetes and non-diabetic controls

\begin{tabular}{lll}
\hline & $\begin{array}{l}\text { Diabetic group } \\
(\mathrm{n}=200)\end{array}$ & $\begin{array}{l}\text { Non-diabetic group } \\
(\mathrm{n}=24)\end{array}$ \\
\hline CMV & $64(32.0 \%)^{*}$ & $2(8.3 \%)$ \\
C pneumoniae & $7(3.5 \%) \dagger$ & $0(0 \%)$ \\
\hline
\end{tabular}

${ }^{*} p<0.001$ in comparison with $C$ pneumoniae, and $p<0.05$ in comparison with non-diabetic group. $†$ Six of the seven $C$ pneumoniae positive cases were also also positive for CMV

\section{RESULTS}

We have developed highly sensitive and specific PCR methods for the detection of the smallest traces of $C$ pneumoniae and CMV in tissue samples. The sensitivity of the nested PCR for CMV was 0.1 plaque forming units. For $C$ pneumoniae, as few as 0.4 inclusion forming units of $C$ pneumoniae DNA could be detected by PCR and visualised by hybridisation of the product on a nylon membrane. There was no significant difference between the sensitivities of the two PCR protocols to detect CMV and $C$ pneumoniae DNA in the samples.

Using PCR, CMV nucleic acid sequences were detected in 64 of $200(32 \%)$ samples and C pneumoniae in seven of 200 (3.5\%) samples of the anterior or posterior tibial artery of patients with diabetes who had undergone surgical amputation for gangrenous lower limbs with severe atherosclerosis. Of those positive for $C$ pneumoniae, six $(85.7 \%)$ were also positive for CMV. In contrast, in the control group, CMV nucleic acid was detected in only two of the 24 arterial tissue samples, and none of them was positive for $C$ pneumoniae (table 2 ). Statistical analysis revealed this difference between the incidences of these two pathogens to be highly significant $(\mathrm{p}<0.05)$.

\section{DISCUSSION}

The higher incidence of atherosclerotic diseases in patients with diabetes than in those without diabetes cannot be explained only by the differences in known risk factors or hyperglycaemia, so that other potential risk factors might contribute to their development. There is an accumulating amount of data indicating that infections may be linked to atherosclerotic disease. There are also several potential underlying mechanisms of infection to augment the atherosclerotic process and contribute to later manifestations of overt clinical disease by facilitating plaque rupture and thrombosis.

CMV is ubiquitous, and the incidence of infection gradually increases with age. More than $90 \%$ of adults have experienced infection with CMV. As shown in a recent report from a blood centre of the Chinese Blood Foundation in southern Taiwan, of the 1800 consecutive sera from blood donors tested, only 150 $(8.3 \%)$ were CMV seronegative. ${ }^{26} \mathrm{CMV}$ antigens in the arteries of young trauma victims were often present in the areas of the arterial wall showing early atheromatous change, which indicates that CMV is involved in the development of arterial wall damage rather than being the result of arterial wall damage. ${ }^{11}$ A strong sero-epidemiological link between CMV infection and atherosclerosis has been suggested. ${ }^{12-15} \mathrm{~A}$ case-control study in which patients who had undergone cardiovascular surgery were compared with a control group of subjects who had not undergone surgery but had similar cholesterol values and epidemiological factors was carried out. In approximately 160 pairs of patients, the prevalence of CMV antibodies was higher in the surgical group than in the control group, and a greater percentage of surgical cases than controls had high titres of anti-CMV antibodies. ${ }^{27}$ In some prospective studies with immunosuppressed patients who had received a cardiac transplant, there was a high correlation between CMV positivity and the development of atherosclerosis in the transplanted heart and decreased survival after a five year follow up. ${ }^{13-15}$ In contrast, several recent studies on CMV infection in non-immunocompromised hosts showed no significant correlation between the development of atherosclerosis and CMV infection. ${ }^{28-30}$ This is an intriguing finding, considering that several previous studies have indicated an association between CMV seropositivity and atherosclerosis. ${ }^{12}$ However, they were mostly defined on the basis of coronary restenosis after atherectomy ${ }^{16}$ or the development of lesions in transplanted hearts when the patients were relatively immunocompromised. ${ }^{13-15}$

Chlamydia pneumoniae is an intracellular Gram negative bacterium that commonly causes respiratory infections in all age groups. ${ }^{31}$ Specific antibodies to $C$ pneumoniae have been found in more than half of the adult population. ${ }^{32}$ Persistent infection is not uncommon after acute respiratory infection with $C$ pneumoniae. ${ }^{33}$ Several observations support the hypothesis that persistent $C$ pneumoniae infection might be a risk factor for vascular disease, comparable in magnitude to the classic risk factors. ${ }^{12}{ }^{17} 34$ Seropositivity for $C$ pneumoniae, but not $\mathrm{CMV}$, was recently reported to be associated with atherosclerosis and increased risk for future cardiovascular disease. ${ }^{35}$ Local $C$ pneumoniae infection has subsequently been detected in $52 \%$ of atherosclerotic lesions, but in only $5 \%$ of control samples of the arterial tissue. ${ }^{12}$ Chlamydia pneumoniae first establishes persistent infection in the lung. Infected lung macrophages might then serve as a vehicle to disseminate the infection and to establish persistent infection in the arterial wall. ${ }^{36}$ Infected monocytes not only directly transmit infection to endothelial cells, but also independently enhance the infectivity of C pneumoniae for endothelial cells; the monocytic stimulation of infectivity with C pneumoniae has also been shown to be specific to endothelial cells. ${ }^{37}$ Pre-existing injury or atheromatous lesions, initiated by other risk factors associated with atherosclerosis, in addition to the presence of monocytes, may be needed to establish infection of $C$ pneumoniae in the endothelial cells. In contrast to CMV, active infection with C pneumoniae usually occurs in immunocompetent individuals.

"The impaired immune response may lead to repeated or continuous $\mathrm{CMV}$ reactivation from its latent status in the vascular wall, resulting to endothelial damage, smooth muscle cell proliferation, and an inflammatory response, thus initiating a process of atherosclerosis"

The biological properties of both CMV and C pneumoniae are consistent with a potential role in the pathogenesis of atherosclerosis. However, the potential role of different infectious agents in the pathogenesis of atherosclerosis might rely on their biological properties and their infectivity to hosts with varying immunological status. As shown in our present study, a high incidence of CMV nucleic acid sequences, but not C pneumoniae, is found in the arterial samples of patients with diabetes and severe atherosclerosis. Most of the samples that were positive for $C$ pneumoniae were also CMV DNA positive. Our data support the hypothesis that CMV, but not $C$ pneumoniae, is involved in the pathogenesis of atherosclerosis in patients with diabetes mellitus. It has been shown that both cellular and humoral immune responses to viral antigens in patients with diabetes mellitus are impaired. ${ }^{38-40}$ As already proposed by others, ${ }^{101141}$ the arterial wall may be a site of latency for CMV, and the impaired immune response may lead to repeated or continuous CMV reactivation from its latent status in the vascular wall, resulting in endothelial damage, smooth muscle cell proliferation, and an inflammatory response, thus initiating a process of atherosclerosis. It may act alone or in synergy with other recognised risk factors. This mechanism might contribute to the high incidence of atherosclerotic diseases in patients with diabetes mellitus. 


\section{Take home messages}

- There was a significantly higher incidence of cytomegalovirus (CMV) nucleic acid in the arterial samples of patients with diabetes

- These results support the notion that CMV is involved in the pathogenesis of atherosclerosis in patients with diabetic mellitus

- Chlamydia pneumoniae did not appear to be involved in the pathogenesis of atherosclerosis in these patients

- The potential roles of different infectious agents in the pathogenesis of atherosclerosis might rely on their biological properties and their infectivity in hosts with varying immunological status

We conclude that latent CMV infection of the arterial wall may be a common occurrence in patients with diabetes mellitus. The ability to reactivate the virus from the latent status leading to a local lytic infection in the vessel wall might result in repeated local inflammatory reactions, eventually leading to vascular injury. This injury, together with other established risk factors, might contribute to the process of atherogenesis. Although our present results do not suggest a direct role for CMV in the pathogenesis of atherosclerosis, determining the presence of CMV infection may be a valuable parameter in the management of patients with diabetes. Further studies are needed to elucidate the factors leading to reactivation of CMV and the development of atherosclerosis as a consequence of a local productive infection.

\section{ACKNOWLEDGEMENTS}

The authors are grateful to J-H Bi and F J-H Lin for invaluable technical assistance. This study was supported by grants NSC88-2314B182A-027 and NSC88-2314-B006-080 from the National Science Council, and 91-B-FA09-2-4 from the Ministry of Education of the Republic of China.

\section{Authors' affiliations}

T-M Lin, H-Y Chen, Department of Medical Technology, College of Medicine, National Cheng Kung University, Tainan, Taiwan W-i Chen, H-L Eng, Department of Pathology, Kaohsiung Medical Center, Chang Gung University and Memorial Hospital, Niao-Sung Hsiang, Kaohsiung, Taiwan

P-W Wang, Department of Internal Medicine, Kaohsiung Medical Center

This study was presented in part as a poster at the 100th General Meeting of the American Society for Microbiology, May 2000, Los Angeles, California, USA.

\section{REFERENCES}

1 Hostetter MK. Handicaps to host defense: effects of hyperglycemia on C3 and Candida albicans. Diabetes 1990;39:271-5.

2 McMahon MM, Bistrian BR. Host defenses and susceptibility to infection in patients with diabetes mellitus. Infect Dis Clin North Am 1995;9:1-9.

3 Folsom AR, Liao F, Szklo M, et al. A prospective study of coronary heart disease in relation to fasting insulin, glucose, and diabetes. Diabetes Care 1997;20:935-42

4 Steiner G. Diabetes and atherosclerosis-a lipoprotein prospective. Diabet Med 1997; 14:S38-44

5 Ross R. The pathogenesis of atherosclerosis-a perspective for the 1990s. Nature 1993;362:801-9.

6 Azar RR, Waters DD. The inflammatory etiology of unstable angina. Am Heart J 1996;132:1101-6.

7 Hansson GK. Immune mechanisms in atherosclerosis. Atherosclerosis 1989;9:567-8

8 Steinberg D, Parthasarathy S, Carew TE, et al. Beyond cholesterol. Modifications of low-density lipoprotein that increases its atherogenicity. N Engl J Med 1989;320:915-24

9 Benditt EP, Barrett T, McDougal JK. Viruses in the etiology of atherosclerosis. Proc Natl Acad Sci U S A 1993;80:6386-9.

10 Melnick JL, Petrie BL, Dreesman GR, et al. Cytomegalovirus antigen within human arterial smooth muscle cells. Lancet 1983;ii:644-7.
11 Yamashirogo HM, Ghosh L, Yang R, et al. Herpesviridae in the coronary arteries and aorta of young trauma victims. Am J Pathol 1988;130:71-9

12 Danesh J, Collins R, Peto R. Chronic infections and coronary heart disease: is there a link? Lancet 1997;350:430-6.

13 Grattan MT, Moreno-Cabraxl CE, Starnes VA, et al. Cytomegalovirus infection is associated with cardial allograft rejection and atherosclerosis. JAMA 1989;261:3561-6.

14 Macdonald K, Rector TS, Braunlan EA, et al. Association of coronary artery disease in cardiac transplant recipients with cytomegalovirus infection. Am J Pathol 1989;64:359-62.

15 Wu TC, Hruban RH, Ambinder RF, et al. Demonstration of cytomegalovirus nucleic acids in coronary arteries of transplanted hearts. Am J Pathol 1992;140:739-47.

16 Zhou YF, Leon MB, Waclawiw MA, et al. Association between prior cytomegalovirus infection and the risk of restenosis after coronary atherectomy. N Engl J Med 1996;335:624-30.

17 Grayston JT. Chlamydia pneumoniae and cardiovascular disease. Cardiologia 1997;42:1145-51.

18 Kuo CC. Demonstration of Chlamydia pneumoniae in atherosclerotic lesions of coronary arteries. J Infect Dis 1993;167:841-9.

19 Moazed TC, Kuo CC, Grayston T, et al. Murine models of Chlamydia pneumoniae infection and atherosclerosis. J Infect Dis 1997;175:88390.

20 Gupta S, Leatham EW, Carrington D, et al. Elevated antibodies, cardiovascular events, and azithromycin in male survivors of myocardial infarction. Circulation 1997;96:404-7.

21 Gurfinkel E, Bozovich G, Daroca A, et al. Randomised trial of roxithromycin in non-Q-wave coronary syndromes: ROXIS pilot study. Lancet 1997:350:404-7.

22 Visseren FL, Bouter KP, Pon M, et al. Patients with diabetes mellitus and atherosclerosis; a role for cytomegalovirus? Diabetes Res Clin Pract 1997;36:49-55.

23 Furness G, Graham DM, Reeve R. The titration of trachoma and inclusion blenorrhoea virus in cell culture. J Gen Microbiol 1960;23:613-19.

24 Kouzarides T, Bankier AT, Satchwell SC, et al. Sequence and transcription analysis of the human cytomegalovirus DNA polymerase gene. J Virol 1987;61:125-33.

25 Campbell LA, Perez Melgosa M, Hamilton DJ, et al. Detection of Chlamydia pneumoniae by polymerase chain reaction. J Clin Microbiol 1992;30:434-9.

26 Lu SC, Chin LT, Wu FM, et al. Seroprevalence of CMV antibodies in a blood donor population and premature neonates in the southern-central Taiwan. Kao Hsiung I Hsueh Ko Hsueh Tsa Chih 1999;15:603-10.

27 Adam E, Melnick JL, Probesfield JL, et al. High level of cytomegalovirus antibody in patients requiring vascular surgery for atherosclerosis. Lance 1987; ii:291-3.

28 Adler SP, Hur JK, Wang JB, et al. Prior infection with cytomegalovirus is not a major risk factor for angiographically demonstrated coronary artery atherosclerosis. J Infect Dis 1998;177:209-12.

29 Hendrix MGR, Dormans PHJ, Kitslaar P, et al. The presence of cytomegalovirus nucleic acids in the arterial walls of atherosclerotic and non-atherosclerotic patients. Am J Pathol 1989;134:1151-7.

30 Melnick JL, Hu C, Burek J, et al. Cyłomegalovirus DNA in arterial wall of patients with atherosclerosis. J Med Virol 1994;42:170-4.

31 Kuo CC, Gown AM, Benditt EP, et al. Detection of Chlamydia pneumoniae in aortic lesions of atherosclerosis by immunocytochemical stain. Arterioscler Thromb 1993;13:1501-4.

32 Grayston JT. Infection caused by Chlamydia pneumoniae strain TWAR. Clin Infect Dis 1992;15:757-63

33 Gnarpe H, Gnarpe J. Increasing prevalence of specific antibodies to Chlamydia pneumoniae in Sweden. Lancet 1993:341:381.

34 Kuo CC, Campbell LA. Is infection with Chlamydia pneumoniae a causative agent in atherosclerosis? Mol Med Today 1998;4:426-30.

35 Fragerberg B, Gnarpe J, Gnarpe H, et al. Chlamydia pneumoniae but not cytomegalovirus antibodies are associated with future risk of stroke and cardiovascular disease. Stroke 1999;30:299-305.

36 Moazed TC, Kuo CC. Grayston JT. Evidence of system dissemination of Chlamydia pneumoniae via macrophages in the mouse. $J$ infect Dis 1998; 177:1322-5.

37 Lin TM, Campbell LA, Rosenfeld ME. Monocyte: endothelial cell co-culture enhances infection of endothelial cells with Chlamydia pneumoniae. J Infect Dis 2000;181:1096-100.

38 Bouter KP, Gmelig Meyling FHJ, Hoekstra JBL, et al. Influence of blood glucose levels on peripheral lymphocytes in patients with diabetes mellitus. Diabetes Res 1992:19:77-80.

39 Diepersloot RJA, Bouter KP, Beyer WEP, et al. Humoral immune response and delayed type hypersensitivity to influenza vaccine in patients with diabetes mellitus. Diabetologia 1987;30:397-400.

40 Diepersloot RJA, Bouter KP, Beek VR, et al. Cytotoxic T-cell response to influenza $A$ subunit vaccine in patients with type 1 diabetes mellitus. Neth J Med 1989;35:68-75.

41 Petrie BL, Melnick JL, Adam E, et al. Nucleic acid sequences of cytomegalovirus in cell cultured from human arterial tissues. J Infect Dis 1987; 155:158-9. 